\title{
Front Matter: Volume 10256
}

, "Front Matter: Volume 10256," Proc. SPIE 10256, Second International Conference on Photonics and Optical Engineering, 1025601 (24 April 2017); doi: $10.1117 / 12.2276625$

SPIE Event: Second International Conference on Photonics and Optical Engineering, 2016, Xi'an, China 


\section{PROCEEDINGS OF SPIE}

\section{Second International Conference on Photonics and Optical Engineering}

\section{Chunmin Zhang \\ Anand Asundi \\ Editors}

\section{4-17 October 2016 \\ Xi'an, China}

\section{Organized by}

Xi'an Jiaotong University (China) - Xi'an Institute of Optics and Precision Mechanics (China) Chinese Academy of Sciences (China) • Xi'an Technological University (China) • Xi' an Institute of Applied Optics (China)

Sponsored by

Shaanxi Optical Society (China) - Optics and Photonics Society of Singapore (Singapore) Shaanxi Provincial Physical Society (China) • Chinese Optical Society (China)

\section{Cooperating Organizations}

Centre for Optical and Laser Engineering, Nanyang Technological University (Singapore) State Key Laboratory of Transient Optics and Photonics (China) - Acta Photonica Sinica (China) • Chinese Laser Press (China) - Light: Science \& Applications (China) • Council Units of Shaanxi Optical Society (China)

Supported by

Ministry of Education of the People's Republic of China (China) - Chinese Academy of Sciences (China) - National Natural Science Foundation of China (China) - Shaanxi Association for Science and Technology (China)

Published by

Part One of Two Parts

SPIE

Volume 10256 
The papers in this volume were part of the technical conference cited on the cover and title page. Papers were selected and subject to review by the editors and conference program committee. Some conference presentations may not be available for publication. Additional papers and presentation recordings may be available online in the SPIE Digital Library at SPIEDigitallibrary.org.

The papers reflect the work and thoughts of the authors and are published herein as submitted. The publisher is not responsible for the validity of the information or for any outcomes resulting from reliance thereon.

Please use the following format to cite material from these proceedings:

Author(s), "Title of Paper," in Second International Conference on Photonics and Optical Engineering, edited by Chunmin Zhang, Anand Asundi, Proceedings of SPIE Vol. 10256 (SPIE, Bellingham, WA, 2017) Seven-digit Article CID Number.

ISSN: 0277-786X

ISSN: 1996-756X (electronic)

ISBN: 9781510610132

ISBN: 9781510610149 (electronic)

Published by

SPIE

P.O. Box 10, Bellingham, Washington 98227-0010 USA

Telephone +1 3606763290 (Pacific Time) · Fax +1 3606471445

SPIE.org

Copyright (c) 2017, Society of Photo-Optical Instrumentation Engineers.

Copying of material in this book for internal or personal use, or for the internal or personal use of specific clients, beyond the fair use provisions granted by the U.S. Copyright Law is authorized by SPIE subject to payment of copying fees. The Transactional Reporting Service base fee for this volume is $\$ 18.00$ per article (or portion thereof), which should be paid directly to the Copyright Clearance Center (CCC), 222 Rosewood Drive, Danvers, MA 01923. Payment may also be made electronically through CCC Online at copyright.com. Other copying for republication, resale, advertising or promotion, or any form of systematic or multiple reproduction of any material in this book is prohibited except with permission in writing from the publisher. The CCC fee code is 0277-786X/17/ \$18.00.

Printed in the United States of America.

Publication of record for individual papers is online in the SPIE Digital Library.

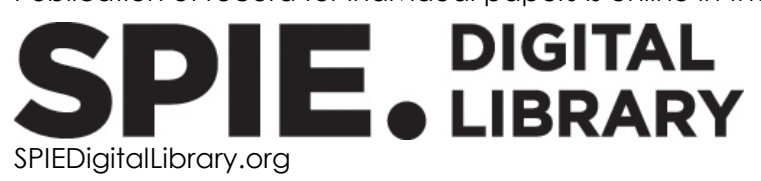

Paper Numbering: Proceedings of SPIE follow an e-First publication model. A unique citation identifier (CID) number is assigned to each article at the time of publication. Utilization of CIDs allows articles to be fully citable as soon as they are published online, and connects the same identifier to all online and print versions of the publication. SPIE uses a seven-digit CID article numbering system structured as follows:

- The first five digits correspond to the SPIE volume number.

- The last two digits indicate publication order within the volume using a Base 36 numbering system employing both numerals and letters. These two-number sets start with 00, 01, 02, 03, 04, $05,06,07,08,09,0 A, 0 B \ldots$. OZ, followed by 10-1Z, 20-2Z, etc. The CID Number appears on each page of the manuscript. 


\title{
Contents
}

\author{
xiii Authors \\ xix Conference Committees \\ xxiii Introduction
}

\section{Part One}

1025602 Design of the optical structure of airfield in-pavement LED runway edge lights [10256-2]

1025603 Predicting the 1s core level spectroscopy of BP using the first principles [10256-3]

1025604 ADRC system of FSM for image motion compensation [10256-4]

1025605 Influence of structural parameters on the laser precision tracking turntable [10256-5]

1025606 Gap solitons in partially parity-time-symmetric optical lattices [10256-6]

1025607 Development of IR imaging system simulator [10256-7]

1025608 Hybrid cylinder-triangle plasmonic waveguide for low loss propagation and subwavelength confinement [10256-8]

1025609 Numerical study of the Bessel beams carrying optical vortices propagating in turbulent atmosphere [10256-9]

10256 OA Terahertz spectral detection of potassium sorbate in milk powder [10256-10]

10256 OB A novel long-range hybrid insulator-metal-insulator plasmonic waveguide with tight light confinement [10256-12]

10256 OC Modal analysis of collimation frame fabricated by titanium alloy [10256-13]

10256 OD Least square support vector machine for citrus greening by use of near infrared spectroscopy [10256-14]

10256 OE Fiber optic grating inscription over splice interface for strain and temperature measurement [10256-15]

$10256 \mathrm{OF}$ Theoretical study on affecting factors on squeezing level in the generation and detection of squeezed light [10256-16]

10256 OG Experimental study on auto-focusing and ranging based on light-field imaging technique [10256-17] 
$10256 \mathrm{OH}$ Design of ocular for optical sight with long exit pupil distance [10256-18]

10256 Ol Full Stokes spectralpolarimeter based on the polarization-difference interference imaging spectrometer [10256-19]

10256 0J Projection matrix acquisition for cone-beam computed tomography iterative reconstruction [10256-20]

10256 OK Fringe projection profilometry based on the best phase sensitivities [10256-21]

$10256 \mathrm{OL}$ Analysis of noise reduction performance with the rotated diffuser in Fizeau interferometer of a large aperture [10256-22]

10256 OM Optical butting of linear infrared detector array for pushbroom imager [10256-23]

1025600 Uncertainty change in length conversion affected by change in environmental parameters [10256-27]

10256 OP Frequency stabilization of DFB laser via modulation transfer spectroscopy [10256-28]

$102560 Q$ Research of the metamaterial on the Chinese map [10256-29]

10256 OR Effects of substrate temperature on properties of vanadium oxide thin films on Si substrate [10256-30]

10256 OS Optical system design with common aperture for mid-infrared and laser composite guidance [10256-31]

10256 OT Imaging through strongly scattering media with subwavelength resolution and good antinoise performance [10256-32]

10256 OU Efficient method of Shack-Hartmann wavefront sensor assembly [10256-33]

10256 OW Influence analysis of satellite drift angle on spectrum recovery precision of large aperture static interferometer spectrometer [10256-35]

10256 OZ Design of linearization double-linkage IR zoom lens [10256-38]

1025610 Optical design of laser zoom projective lens with variable total track [10256-39]

1025613 The design of common aperture and multi-band optical system based on day light telescope [10256-42]

1025614 Parallel transformation of K-SVD solar image denoising algorithm [10256-43]

1025615 The infrared bands Pechan prism axis parallel detection method [10256-44]

1025617 Structural optimization of the path length control mirror for ring laser gyro [10256-46]

1025619 Simulation design of light field imaging based on ZEMAX [10256-48] 
10256 lA Comparison of monomode $\mathrm{KTiOPO}_{4}$ waveguide formed by $\mathrm{C}^{3+}$ ion implantation and $\mathrm{Rb}^{+}$ ion exchange [10256-49]

10256 1B Influence of photoelectric detector on the dynamic range of laser seeker [10256-50]

10256 1C An adaptive Gamma method for image under non-uniform illumination [10256-51]

10256 1E Principle component analysis based hyperspectral image fusion in imaging spectropolarimeter [10256-53]

10256 IF Ultra-short wavelength operation in Thulium-doped silica fiber laser with bidirectional pumping [10256-54]

$102561 G$ Spectrum analysis of radar life signal in the three kinds of theoretical models [10256-56]

$102561 \mathrm{~J}$ A new method named as Segment-Compound method of baffle design [10256-59]

10256 1K Dual-wavelengths photoacoustic temperature measurement [10256-60]

10256 1L The propagation characteristics of circular Airy beam with low-pass filtering modification [10256-61]

$102561 \mathrm{~N} \quad$ Automatic phase aberration compensation and imaging of digital holographic microscopy [10256-65]

1025610 Fast recovery method for fog image [10256-67]

$102561 Q \quad$ Optical humidity detection based on tunable diode laser absorption spectroscopy [10256-69]

10256 IR Numerical simulation and experimental research of using spherical reference wavefront to test aspheric surface [10256-70]

10256 is Numerical analysis of thermally tunable liquid-crystal-filled terahertz fiber [10256-71]

$102561 \mathrm{E} \quad \mathrm{Er}^{3+}$ doped $\mathrm{Pb}\left(\mathrm{Mg}_{1 / 3} \mathrm{Nb}_{2 / 3}\right) \mathrm{O}_{3}-0.25 \mathrm{PbTiO}_{3}$ transparent ceramic: a multi-functional material for photonics switching and temperature measurement [10256-74]

10256 IV Research on electromechanical resonance of two-axis tracking system [10256-75]

10256 IW Inter-satellites x-ray communication system [10256-76]

$102561 \mathrm{X}$ Optimal design of an earth observation optical system with dual spectral and high resolution [10256-77]

10256 IY Study of fuzzy PID controller for velocity circuit of optical-electronic theodolite [10256-80]

1025612 A micro displacement measurement method based on PGC demodulation of space optics [10256-81]

1025622 Calibration on the detection efficiency of the Si-APD and InGaAs-APD single-photon detectors by correlated photon pairs [10256-84] 
1025623 Design of the intelligent smoke alarm system based on photoelectric smoke [10256-85]

1025624 Mechanism and experimental research on ultra-precision grinding of ferrite [10256-86]

1025625 Movement decoupling control for two-axis fast steering mirror [10256-87]

1025626 Analysis and compensation of disturbance for small inertial stabilized line-of-Sight system [10256-88]

1025627 Research of the wavefront detection method based on the scanning pentaprism [10256-89]

1025629 Control of laser wavelength tuning and its application in coherent optical time domain reflectometer [10256-91]

10256 2A The analysis of bit error ratio for self-homodyning coherent detection system [10256-92]

10256 2C Influence study of parameters to holographic interference pattern by computer simulation [10256-94]

10256 2D Study of thin film thickness measurement based on white light interference [10256-95]

$102562 \mathrm{E}$ Acceleration sensing based on graphene resonator [10256-96]

$102562 F \quad$ FPGA implement method for two-dimensional integer wavelet transform in the space-based on-orbit image compression system [10256-97]

$102562 \mathrm{H}$ The cooling control system for focal plane assembly of astronomical satellite camera based on TEC [10256-99]

1025621 Design and analysis of micro stress flexible support structure of reflector in all day star tracker [10256-100]

10256 2J Improved image quality of digital lithography using modified particle swarm optimization algorithm [10256-101]

10256 2K A dual-direction fringe projection method for the 3D measurement of translucent object [10256-102]

10256 2L The 3D measurement techniques for ancient architecture and historical relics [10256-103]

$102562 \mathrm{~N}$ Investigation of grinding parameters and machine dynamic characteristics' effect towards brittle material subsurface damage [10256-105]

1025620 High-accuracy measurement and compensation of grating line-density error in a tiled-grating compressor [10256-106]

1025625 Dispersion characteristics of Tellurite glass photonic crystal fiber [10256-110]

$102562 \mathrm{~T}$ Accelerating hyper-spectral data processing on the multi-CPU and multi-GPU heterogeneous computing platform [10256-111] 
$102562 \mathrm{U}$ Research on spot of CCD subdivided locating methods in laser triangulation displacement measurement [10256-112]

$102562 \mathrm{~V}$ Analysis of a novel device-level SINS/ACFSS deeply integrated navigation method [10256-113]

10256 2W A compact LWIR imaging spectrometer with a variable gap Fabry-Perot interferometer [10256-114]

10256 2X A Fabry-Perot interferometric imaging spectrometer in LWIR [10256-115]

$102562 Y$ Application of linear CCD in tunnel crack detection [10256-117]

$102562 Z$ A reflection polarizations zoom metasurfaces [10256-118]

1025630 Synchronous acquisition method based on feature recognition of sequence images in online vision inspection [10256-119]

1025632 Optimized design method for trench-assisted grade-index ring-core fiber with low DMD and large $A_{\text {eff }}[10256-123]$

1025633 Dual-band perfect absorbers based on the magnetic resonance and the cavity resonance [10256-124]

1025634 Evolution of the Raman spectra of electron beam irradiated graphene exposed in air [10256-125]

1025635 A novel design of subminiature star sensor's imaging system based on TMS320DM3730 [10256-126]

\section{Part Two}

1025636 Automatic seamless image mosaic method based on SIFT features [10256-127]

1025637 Intelligent large diameter aspherical reflector polishing technology [10256-128]

1025639 Fourier transform profilometry based on mean envelope extraction [10256-130]

$102563 \mathrm{~A}$ Atmospheric transmittance of $\mathrm{O}_{2}(0-1)$ airglow [10256-131]

10256 3C Improving the atmospheric wind speed measured accuracy by the ground-based airglow imaging interferometer [10256-133]

10256 3E Design of laser Michelson interferometer for the detection of nanolitre solution [10256-136]

10256 3F High voltage pulse gated power supply with adjustable pulse width [10256-137]

$102563 \mathrm{H} \quad$ Research on transmission high sensitivity GaAs cathode of low light level image devices [10256-139] 
1025631 Effect of the characteristics of beam polarization on performance of 90 degree optical hybrid [10256-140]

$102563 \mathrm{~J} T$ Theoretical and experimental analysis of modern zoom lens design [10256-141]

10256 3K A novel approach of an absolute coding pattern based on Hamiltonian graph [10256-143]

$102563 \mathrm{~L}$ Method of improving photoelectric efficiency for laser power beaming based on photovoltaic cell layout optimization [10256-144]

$102563 \mathrm{M}$ The design of fiber-based 4-LP mode multi/demultiplexer [10256-145]

10256 3N Precision glass molding technology for low Tg glasses [10256-146]

1025630 Femtosecond laser ablated durable superhydrophobic PTFE sheet for oil/water separation [10256-147]

10256 3P Ray-tracing analysis of a snapshot imaging polarimeter using modified Savart polariscopes [10256-148]

$102563 Q$ Fabrication of the asymmetric double-sided concave microlens arrays by femtosecond laser [10256-149]

1025635 Fabrication of 3D electro-thermal micro actuators in silica glass by femtosecond laser wet etch and microsolidics [10256-151]

10256 3T Micropatterning microlens arrays fabricated by a femtosecond laser wet etch process [10256-153]

$102563 \mathrm{~V}$ Study on the material quenching over $\mathrm{ZnS}: \mathrm{Cu}$ and $\mathrm{SrAl}_{2} \mathrm{O}_{4}: \mathrm{EU}^{2+}, \mathrm{DY}^{3+}$ by the infrared light [10256-155]

10256 3W Power build-up cavity enhanced Raman spectroscopy based on piezoelectric transducer for gas analysis [10256-156]

$102563 X$ Athermalization of infrared dual field optical system based on wavefront coding [10256-157]

$102563 Y$ Study on general design of dual-DMD based infrared two-band scene simulation system [10256-159]

$102563 Z$ Design of a common-aperture VIS/LWIR imaging optical system with muti-field of view [10256-160]

1025640 Host-guest interaction between Acridine orange molecules and AFI or CHA zeolite crystals [10256-161]

1025641 The development of 4-channel Fourier transform polarization spectrometer [10256-162]

1025642 Fourier modal method for two-dimensional wavefront reconstruction [10256-163] 
1025643 Design to improve photoelectric efficiency for photovoltaic cell array for laser power beaming [10256-165]

1025644 A vision-based fall detection algorithm of human in indoor environment [10256-166]

1025645 Study on light scattering characterization for polishing surface of optical elements [10256-168]

1025648 A division-of-wave-front photopolarimeter for the measurement of the polarization state of light [10256-175]

1025649 A novel experimental mechanics method for measuring the light pressure acting on a solar sail membrane [10256-176]

10256 4A Research on key technology in the real-time and high-precision spot centroid detection [10256-177]

10256 4B Study on the optical properties of the off-axis parabolic collimator with eccentric pupil [10256-178]

10256 4C Polarization imaging of an edge object with partially coherent light [10256-179]

10256 4D Research on environment correction algorithm in the minimum deviation angle method for refractive index measuring [10256-180]

$102564 \mathrm{~F}$ Simulation of the fixed optical path difference of near infrared wind imaging interferometer [10256-182]

$102564 \mathrm{G}$ Methods of degrading the polarization sensitivity of remote sensing cameras for ocean exploration [10256-183]

$102564 \mathrm{H}$ An estimated method of visibility for a remote sensing system based on LabVIEW and Arduino [10256-184]

10256 4K Review of $1064 \mathrm{~nm}$ single frequency fiber laser based on different saturable absorber [10256-187]

10256 4L Study on the key alignment technology of the catadioptric optical system [10256-188]

$102564 \mathrm{M}$ Experimental research for relative radiometric calibration of imaging spectrometer based on Savart plates [10256-189]

10256 4P Design of VisSWIR continuous zoom optical system [10256-192]

$102564 \mathrm{Q}$ Research on the influence of the vertical temperature profile on the retrieval of $\mathrm{CO}_{2}$ concentration [10256-193]

$102564 \mathrm{~V}$ Comparison of temporal phase-stepping measurement methods used for wind imaging interferometer [10256-198]

10256 4W Low-power and precise temperature control for high-power CCD assembly [10256-199] 
$102564 \mathrm{X}$ Aspheric surface measurement using capacitive probes [10256-202]

$102564 Z$ Crystal structure and optical properties of a neodymium trifluoroacetate complex for liquid laser [10256-205]

1025650 Discuss wave-particle duality of light [10256-207]

1025651 Design of a handheld infrared imaging device based on uncooled infrared detector [10256-208]

1025652 Research on the effect of aerosol on the retrieval of hyperspectral XCO 2 [10256-209]

1025653 He-Ne laser employing radio frequency for pumping of gain medium [10256-210]

1025654 Discussion on method of optical surface roughness measurement [10256-211]

1025655 Method and verification for measuring surface roughness of components by angle resolved scattering method [10256-212]

1025656 Terahertz range profile of the tilted-plate [10256-213]

1025657 Global optimization method based on ray tracing to achieve optimum figure error compensation [10256-215]

1025658 Numerical simulation of optical interference for double elliptically polarized beams [10256-218]

1025659 Plasma etching of large-size silicon based microchannel plates [10256-219]

$102565 \mathrm{~A}$ Application of fluorescence spectroscopy and imaging in the detection of a photosensitizer in photodynamic therapy [10256-221]

10256 5B Optical properties of a paramagnetic metalloporphyrin hematoporphyrin monomethyl ether coordinated to divalent manganese metal ion [10256-225]

$102565 \mathrm{C}$ Short-wave infrared imaging technology on space optical remote sensing system [10256-226]

$102565 \mathrm{D}$ The influence of temperature and pressure on primary mirror surface figure and image quality of the $1.2 \mathrm{~m}$ colorful Schlieren system [10256-1001]

$102565 \mathrm{E} \quad$ Numerical simulation of radiation intensity of a long-endurance UAV exhaust system with 2D nozzle [10256-1002]

$102565 \mathrm{G}$ Photonic generation of frequency quadrupling signal for millimeter-wave communication utilizing three parallel Mach-Zehnder modulators [10256-1004]

$102565 \mathrm{H} \quad$ A polyatomic photonic crystal ring resonator and its application to the optical biochemical sensor [10256-1005]

1025651 Simultaneous measurement of temperature and strain based on a fiber Bragg grating with cladding made of electro-optic crystal material [10256-1006] 
10256 5J Study of the fusion point between PM-PCF and panda fiber and its influence to interferometric fiber optical gyroscope [10256-1007]

$102565 \mathrm{M} \quad$ Numerical study of the light output intensity of the bilayer organic light-emitting diodes [10256-1010]

$102565 \mathrm{~N}$ A combination of JND curve property and intensity-pair distribution for image enhancement [10256-1011]

$1025650 \quad$ ZnS:Co film grown by pulsed laser deposition and optical properties analysis [10256-1012]

10256 5P Operational data quality evaluation based on BP neural network and rough set theory [10256-1013]

10256 5R Programmable spectral engine design of hyperspectral image projectors based on digital micro-mirror device (DMD) [10256-1015]

$102565 S$ Study of femtosecond laser spectrally resolved interferometry distance measurement based on excess fraction method [10256-1016]

$102565 \mathrm{~T}$ Theoretical and experimental investigation on superconducting nanowire single-photon detectors [10256-1017]

$102565 \mathrm{U}$ System design for adopting magnetofluid and LPFG to measure magnetic field and temperature [10256-1018]

10256 5V Design of edge filter demodulation system based on twin-core LPFG [10256-1019]

10256 WW Research on alignment between processing and testing coordinates in flat mirror manufactory [10256-1020]

10256 5X Study and analysis on slow light in photonic crystal waveguide [10256-1021]

$102565 Y \quad$ Effect of polarizer parameters on measuring Verdet constant of magneto-optical glass [10256-1022]

$102565 Z$ Simulation and design of solar-blind Raman Lidar for water vapor measurement [10256-1023]

1025660 Infrared scene projector optical system design with wide field-of-view [10256-1024]

1025661 Polarization insensitive and low-loss coupling mode-size converter from super luminescent diode to silica-based planar lightwave circuit [10256-1025] 
Proc. of SPIE Vol. 10256 1025601-12 Downloaded From: https://www.spiedigitallibrary.org/conference-proceedings-of-spie on 26 Apr 2023
Terms of Use: https://www.spiedigitallibrary.org/terms-of-use 


\section{Authors}

Numbers in the index correspond to the last two digits of the seven-digit citation identifier (CID) article numbering system used in Proceedings of SPIE. The first five digits reflect the volume number. Base 36 numbering is employed for the last two digits and indicates the order of articles within the volume. Numbers start with 00, 01, 02, 03, 04, 05, 06, 07, 08, 09, OA, OB...0Z, followed by 10-12, 20-2Z, etc.

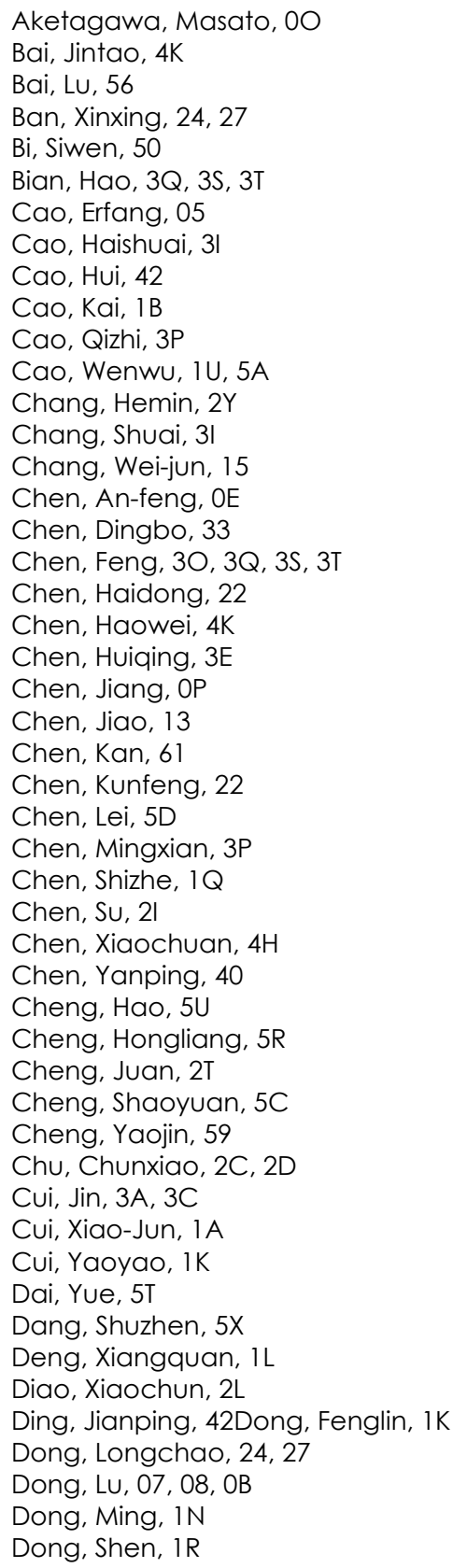

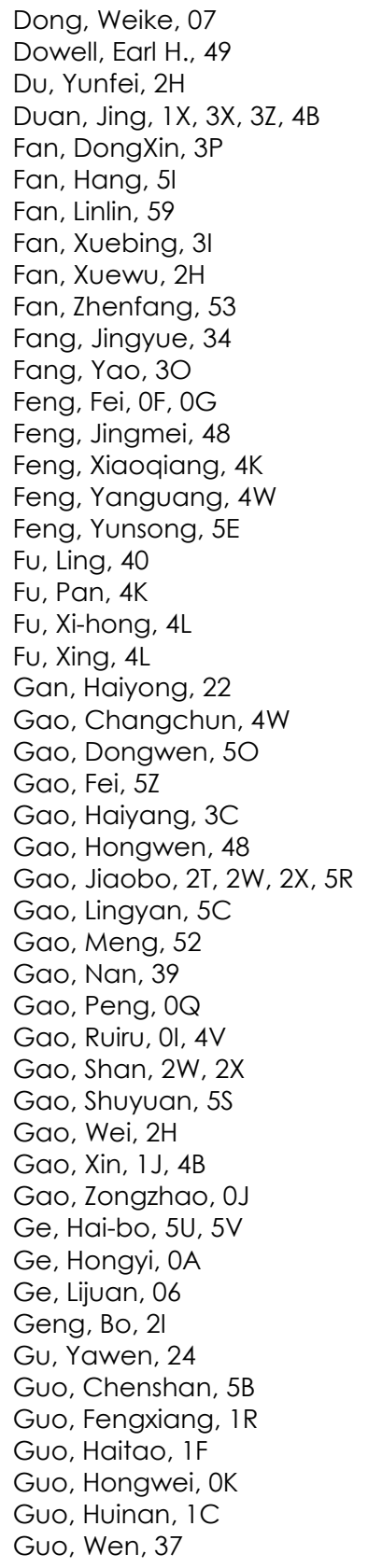


Guo, Xuejia, 57

Guo, Yongcai, 44

Guo, Youguang, 12

Han, Junfeng, 26

Han, Kunye, OS, 10, 3H

Han, Rubing, OD

Han, Zonghu, 17

Hang, Nan, 3l

Hao, Fusheng, 3K

Hao, Juan, 1B

$\mathrm{He}$, Guojing, 07

He, Junfeng, $5 \mathrm{~N}$

$\mathrm{He}$, SaiXian, $1 \mathrm{Y}$

He, Yulan, 0Z, 10, 15

$\mathrm{He}$, Yuqing, $2 \mathrm{H}$

Hou, Dianxin, OR

Hou, Lei, 4K

Hou, Shanglin, 5G, 5H, 5

Hou, Xun, 3S

$\mathrm{Hu}, \mathrm{BO}, \mathrm{OS}$

Hu, Feng, 2E

$\mathrm{Hu}$, Guixiang, 5B

$\mathrm{Hu}$, Jialin, 3W

Hu, Kun, 5S

Hu, Shi Y., OG

$\mathrm{Hu}$, Xiaoying, 4C

$\mathrm{Hu}, \mathrm{Yu}, 2 \mathrm{~T}$

Hua, Dengxin, $5 Z$

Huang, Jie, 33

Huang, Kuidong, $0 \mathrm{~J}$

Huang, Qiaolin, 5C

Huang, Shujun, 39

Huang, Yuhua, 42

Huang, Zhang-bin, 5E

Huang, Zhihao, 34

Hui, Lei, 2U

Huo, Jinglan, 30

Ji, Ming, 15

Ji, Rongyi, 5S

Jia, Min, 3A, 3V

Jia, Xiaoyang, 2A

Jian, Dunliang, 40

Jian, Xiaohua, $1 \mathrm{~K}$

Jiang, Bo, 3X

Jiang, Guangwen, 2V

Jiang, Hongzhi, 2K, 2L

Jiang, Jiangang, $1 \mathrm{E}$

Jiang, Kai, IX, 3X, $3 Z$

Jiang, Li, 49

Jiang, Man, 4K

Jiang, Mingzhu, $2 S$

Jiang, Shubo, 3W

Jiang, Wei, 5C

Jiang, Xiaobai, $\mathrm{OZ}$

Jiang, $X i n, 2 Y, 30$

Jiang, Yongliang, 5W

Jie, Wenbin, 2E

Kang, Jin, 4K

Kang, Wenli, $\mathrm{OZ}$

Kang, Xiao-peng, 4L
Lang, Jia-Wei, $5 \mathrm{U}$

Lei, Jingli, 5G

Lei, Ning, $5 Z$

Li, B., $2 \mathrm{~N}$

Li, Baihong, IS

Li, Baopeng, $2 \mathrm{H}$

Li, Baoquan, IW

Li, Bei, 61

Li, Beibei, 3L, 43

Li, Binglin, 29

Li, Gang, 3Z, 4B

Li, Gengxin, IY

Li, Guorong, $1 \mathrm{U}$

Li, Hong, 2K

Li, Hua, $2 Y, 30$

$\mathrm{Li}$, Irene Ling, 40

$\mathrm{Li}$, Jianjun, 5R

Li, Junwei, 51, 5J

$\mathrm{Li}, \mathrm{Ke}, 61$

Li, Lei, 02

Li, Liangfu, $5 \mathrm{~N}$

Li, Mei, 14, 48

Li, Pengpeng, OA

Li, Qiang, 5W

Li, Qichao, 35

Li, Qishen, 2J

Li, Qiwei, OI, 4M

Li, Shaobo, 4X

Li, Shaohua, 3E

Li, Shufeng, 50

Li, Siyuan, OW

Li, Wentao, 30

Li, Xiaojiang, 3L, 43

Li, Xiaoman, 4G

Li, Xiao-xia, 5E

Li, Xiaoyan, 2A

Li, Xuanqun, $1 Q$

Li, Xun, OT

Li, Yanfen, 4F, 4Q

Li, Yanhui, 56

Li, Yao, $5 \mathrm{~S}$

Li, YU, 2T

Li, Yuan, 10

$\mathrm{Li}$, Yuyao, $\mathrm{OH}$

$\mathrm{Li}$, Zhe, 04

Li, Zhiguo, OC, IV

Li, Zhilin, 20

Lian, Xinhao, 4W

Liang, Peiying, 42

Liang, Xiaoyue, 2K

Liang, Youwen, 14

Liang, Zhang, $1 \mathrm{U}$

Liao, Jiawen, 1C, 10

Liao, Yu, 1K

Lin, Ling, 1B

Liu, Bingcai, OL, 27

Liv, Changming, 22

Liu, Cheng, 61

Liu, Dandan, OP

Liu, Deli, OD

xiv

Proc. of SPIE Vol. 10256 1025601-14 
Liv, Dong, $5 \mathrm{~W}$

Liu, Dongdong, 4F, 4Q

Liu, Hao, 44

Liu, Hongxia, 08, OB

Liu, Huan, OW

Liu, Huan, 59

Liu, Jie, 2I, 2Y, 30

Liu, Jing, 58

Liu, Kai, 1X, 3X, 3Z, 4L

Liu, Kun, 3E

Liu, Li, 15

Liu, Lijun, 5P

Liu, Liqiang, 3K

Liu, Meiying, 35, 36

Liv, Shixuan, $1 Q$

Liv, Shulin, 3F

Liu, Tong, $2 \mathrm{~F}$

Liu, Weiguo, 45

Liu, Weilin, 3J

Liv, Xiaolin, 57

Liu, Xishuang, $1 \mathrm{~J}$

Liu, Xuelian, 54

Liu, Yande, OD

Liu, Yang, 21

Liu, Yanjun, 5G, 5H, $5 \mathrm{I}$

Liu, Zhaohui, OC

Liu, Zun L., OF, OG

Long, Keping, 32, 3M

Long, Xingwu, $1 \mathrm{Z}$

LU, Baole, 4K

Lu, Feiping, 5M

LU, Feng, 10

Lu, Guangfeng, 53

Lü, Hao, 58

Lu, Lidong, 29

LU, Min, IF

LU, Yang, 5N

LU, YUan, OR

Luan, Yadong, OU, 19

Luo, Hui, 53

Luo, Xiaohe, 4A

Luo, Yangcheng, 3E

LV, Dongmei, 54

Lv, Tao, 25

$\mathrm{Ma}$, Congcong, $\mathrm{OB}$

Ma, Donglin, 09

Ma, HongWei, $1 \mathrm{~N}, 1 \mathrm{~s}$

Ma, Jiangfei, 1G, 23

$M a$, Jing $T$., OF, OG

Ma, Junwei, IB

Ma, Tingting, 2A

Ma, Wenpo, OM

Ma, Xiaodan, 02

Ma, Yanghua, 17

Mei, Chao, 4P

Mei, Yifeng, 3A, 3V

Meng, Hemin, $2 X$

Meng, Xiangsheng, 2F

Mou, Huan, IW

$\mathrm{Mu}$, Hang, 5G, 5H
Mu, Jie, 20

$\mathrm{Mu}$, Tingkui, $\mathrm{Ol}$

Nie, Rongzhi, $4 Z$

Pan, Yue, 3Y, 60

Peng, Bo, 1F, $4 Z$

Peng, Jun, 02

Peng, Xiangyang, 34

Qi, Xinyuan, 4K

Qian, Jun, 5N

Qiang, Hua, 0Z, 10, 15

Qiao, Xihui, 32

Qiao, Yang, 3Y, 60

Qiao, Yongming, 25

Qin, Shiqiao, 2E, 2V

Qin, Xing, 1J

Qin, Zhixuan, 49

Qiv, Baowei, 37

Qiv, Minpu, OM

Qu, Rui, 4P

Qu, Sheng, 08, OB

Quan, Bingxin, 17

Quan, Naicheng, 01

Ren, D. X., 2N

Ren, Dong, $5 \mathrm{H}$

Ren, Wen-yi, OE, $1 \mathrm{E}$

Rong, Piao, 4F, 4Q

Ruan, Chi, 4H

Ruan, Jun, OP

Ruan, Shuangchen, 40

Shan, Chao, 3S

Shan, Qiu-sha, 1X, 3X, 3 Z

Shao, Xinzheng, $3 \mathrm{~N}$

Shao, Yajun, 4A

She, Jiangbo, $4 Z$

She, Wenji, 05

She, Xuan, 61

Shi, Aiming, 49

Shi, Dongchen, $5 z$

Shi, Hongwei, 54, 55

Shi, Junru, OP

Shi, Wenlong, OJ

Shi, Xueshun, 22

Shi, Yaotao, 5W

Shi, Zhaojun, 2J

Shu, Jing, $5 X$

Shu, Xiaowu, 61

Song, Chong, 4L

Song, Fuyin, OR

Song, Xinyang, $4 \mathrm{~W}$

Su, Jingqin, $2 \mathrm{O}$

Su, Xiuqin, 04

Sun, Chuan, 4D

Sun, Dandan, 2T

Sun, Kefeng, 2T

Sun, Tao, IR

Sun, Ting, OS

Sun, Xianzhong, 51

Sun, Xiaoyan, 29

Sun, Xin, $4 Z$

Sun, Xudong, OD 
Sun, Zuoming, 5J

Tam, Wing Yim, 58

Tan, Wenfeng, $2 \mathrm{~V}$

Tang, Tianjin, 57

Tang, Yuanhe, 3A, 3C, 3F, 3V

Tao, Xin, 4X

Teng, Guoqi, 13

Tian, Ailing, $\mathrm{OH}, \mathrm{OL}, 27,45,4 \mathrm{C}$

Tian, Chengjun, 55

Tian, Minqiang, $3 \mathrm{~N}$

Tian, Yan, 2F

Tian, Yanan, 27

Tian, Yu, 14

Ting, Deng, 3P

Tong, Shoufeng, 2A, 31

Tu, Jiajing, 32, 3M

Wang, Chunhui, 45

Wang, Chunyang, 54, 55

Wang, D., $1 G$

Wang, Daobin, 5G, 5H, $5 \mathrm{I}$

Wang, Dasen, 45

Wang, Doudou, iN, is

Wang, Haotian, $1 \mathrm{~N}$

Wang, Hongjun, $\mathrm{OL}, 2 \mathrm{U}$

Wang, Hongqing, 33

Wang, Hu, 2l, 35

Wang, Huawei, 3K

Wang, Hushan, IF

Wang, Jian, OE

Wang, Jie, $2 F$

Wang, Jiliang, 17

Wang, Jing, 5D

Wang, Jingyi, 4G

Wang, Kaidi, 04

Wang, Li, $5 \mathrm{O}$

Wang, Li, $5 Z$

Wang, Liang-Ling, 1 A

Wang, Lili, 1S

Wang, Ling, 13, 15

Wang, Lingguang, 21

Wang, Meng, 13

Wang, Nan, 2W, 2X

Wang, Nanxi, 0Z, 10, 15

Wang, Peipei, 23

Wang, Peng, 37

Wang, Peng, 5D

Wang, Qiwei, 1R

Wang, Rongli, 1B

Wang, Rui, 25

Wang, Shanshan, 4A, 4D, 59

Wang, Shuhua, 5J

Wang, Shulong, 08, OB

Wang, Wei, 41, 4C

Wang, Wen, $1 \mathrm{R}$

Wang, Wenyan, 1Q

Wang, Xia, 2C, 2D, 58

Wang, Xiangyang, 3J

Wang, Xiao, 20

Wang, Xiaoyan, $2 Z$

Wang, Xicheng, 5R
Wang, Xing, $1 \mathrm{~N}$

Wang, Xingshu, 2E, 2V

Wang, Xinliang, OP

Wang, Ya'nan, 3K

Wang, Yinghui, 2T

Wang, Yishan, $1 \mathrm{~F}$

Wang, Yu, 48

Wang, Yulei, 5W

Wang, Zefeng, 1C, 10

Wang, Zhibin, $3 \mathrm{~N}$

Wei, Dong, 00

Wei, Peiwen, 5V

Wei, Yang, 3T

Wen, Desheng, 35, 36

Wen, Kaile, 3F

Wu, Dan, 1E

Wu, Jianghui, $2 X, 5 R$

Wu, JunFang, 03

Wu, Lei, 08, OB

Wu, Qi, OE

Wu, Qingyang, $1 \mathrm{~L}$

Wu, Shaobo, 04

Wu, Wei, 2V

Wu, Wenjun, 33

Wu, Y. Y., 2N

Wu, Yushang, IQ

Wu, Zhensen, 56

$\mathrm{Xi}$, J. P., $2 \mathrm{~N}$

Xia, Jianpei, 42

Xiang, Xinglang, 07, 08

Xiao, Gongli, 33

Xiao, Huaichun, OD

Xiao, Xiangguo, 10, 19

Xiao, Xusheng, $1 \mathrm{~F}$

Xie, Yong-jun, 5D

Xin, Ruihao, 54, 55

Xiong, Baoxing, 2S, $5 Y$

Xiong, Zhenyu, $1 \mathrm{Z}$

$X U$, Jiang $M$., OF, OG

$X U$, Jiangtao, $3 \mathrm{H}$

$\mathrm{Xu}, \mathrm{Ke}, 3 \mathrm{H}$

$X \cup$, Shixiang, $1 \mathrm{~L}$

$X u$, Songbo, 5D

$X U$, Weicai, 5W

$X U$, Xintong, 40

$X U$, Xinyang, 60

$X U, X i-p i n g, 3 Y, 60$

$\mathrm{XU}$, Yanhua, 3P

$\mathrm{Xu}$, Yantao, $\mathrm{IF}$

Xue, Yingjie, IV

Yan, Baojun, 3F

Yan, Haozheng, 5N

Yan, Lisong, 5W

Yan, Peipei, 1X, 3X, $3 Z$

Yan, Peng, 33

Yan, Tingyu, Ol, 4F, 4M, 4V

Yan, Zhijun, $1 \mathrm{~F}$

Yang, Fulong, $2 Z$

Yang, Fuqiang, $0 \mathrm{~J}$

Yang, Guoan, $1 \mathrm{E}$ 
Yang, Hong, 3N

Yang, Hongtao, 10,4P

Yang, Huamei, OS

Yang, Jianhong, 02

Yang, Jing, 15

Yang, Junbo, 33

Yang, Ming, 4W

Yang, Mingyang, 4P

Yang, Qing, 3O, 3Q, 3S, 3T

Yang, Rui, 3C

Yang, Shaodong, 35

Yang, Wengang, $2 \mathrm{H}$

Yang, XiaoJun, $1 Y$

Yang, Xiaoxu, $1 \mathrm{~J}$

Yang, Xiufang, 1G, 23

Yang, Yizhou, 5N

Yang, Yong, 5V

Yang, Yongying, 5W

Yang, Yuzhen, 3F

Yang, Zijian, OS

Ye, Lingyu, OD

Yong, Jiale, 30

You, Kai, 58

Yu, Fengxiang, OP

Yu, Xudong, $1 Z$

Yu, Yang, 3A, 3C, 3F

Yuan, Daocheng, 27, 4X

Yuan, Lihua, $5 \mathrm{H}$

Yuan, Xiao, 2S, 5Y

Yuan, Xiuhua, 09

Zang, Lixin, 5A, 5B

Zeng, Shuqin, $1 \mathrm{~V}$

Zeng, Xiaoming, 20

Zhai, Fengtao, 3A, 3C

Zhai, Jianpang, 40

Zhang, Bo, 13

Zhang, Caixin, OJ

Zhang, Chunmin, Ol, 1E, 48, 4F, 4M, 4Q, 4V, 52

Zhang, Chupeng, 24

Zhang, Dinghua, OJ

Zhang, Fan, $3 Q$

Zhang, Fang, 2W, 2X

Zhang, Feng, 3N

Zhang, Fengli, IR

Zhang, Geng, OW

Zhang, Guiju, 2S, 5Y

Zhang, Haifeng, 1C, 10

Zhang, Hao, 2V

Zhang, Henjin, 4B

Zhang, Huan, $3 M$

Zhang, Hui, OP

Zhang, Hui, 10

Zhang, Jiaying, OL, 2U

Zhang, Jie, 5U

Zhang, Jing, 3P

Zhang, Jingjing, 33

Zhang, Keke, IQ

Zhang, Lei, 2T, 2W, 2X

Zhang, Liang, 2J

Zhang, Lijuan, 1Q
Zhang, Meng, 26

Zhang, Peng, 31

Zhang, Qi, 1B

Zhang, Rui, 1B

Zhang, Ruihua, OK

Zhang, Shougang, OP

Zhang, Shuanmin, $\mathrm{OE}$

Zhang, Xiaoxuan, 39

Zhang, Xin, 53

Zhang, Xiumei, 3W

Zhang, Xuanzhi, OS

Zhang, Xuao, 34

Zhang, Xunzhi, OU

Zhang, Yalin, 09

Zhang, Yazhou, 51

Zhang, Yingge, 45

Zhang, Yishu, $1 \mathrm{~N}$

Zhang, Yongqiang, OC

Zhang, Yuan, OA

Zhang, Yunlong, 3N

Zhang, Yupeng, $5 Y$

Zhang, Zhi, 1C, 10

Zhang, Zhiguo, 1U, 5A

Zhang, Zhoufeng, OW

Zhang, Zonghua, 39

Zhao, Dan, 20

Zhao, Huijie, 2K, 2L

Zhao, Huimin, 5A, 5B

Zhao, Huiying, 24, 27

Zhao, Juan, 41

Zhao, Kun, 22

Zhao, Liang, 55

Zhao, Qiang, OW

Zhao, Qiang, 1Q

Zhao, Qiuling, 58

Zhao, Yujie, $2 \mathrm{~W}$

Zhao, Z. X., 2N

Zhao, Zhanping, 5C

Zhao, Zhiming, $1 \mathrm{~V}$

Zhao, Zhiyong, $2 \mathrm{~L}$

Zhao, Zongzhe, 1B

Zheng, Guoliang, IL

Zheng, Hairong, 4H

Zheng, Xiaoming, 34

Zheng, Ye-liang, 5E

Zheng, Yuanbo, 2C, 2D

Zhou, Feng, 4G

Zhou, Guoliang, 5N

Zhou, Kainan, 20

Zhou, Ke, OU, 19

Zhou, Liang, 05

Zhou, Siyu, 4A, 4D

Zhou, Song, 20

Zhou, Weihu, $5 S$

Zhou, Xiaobin, OU, 19

Zhou, Yueming, 52

Zhou, Yulan, OT

Zhou, Zeyu, 09

Zhou, Zhonghai, $1 \mathrm{R}$

Zhou, Zuofeng, 4P 
Zhu, Changyong, 3E

Zhu, Qihua, 20

Zhu, Qiudong, 4A, 4D

Zhu, Xueliang, OL, 24, 27

Zhu, Zhongyao, $\mathrm{OH}$

Zou, Bin, $5 \mathrm{~N}$

Zou, Kuaisheng, 2S, $5 Y$

Zuo, Yanlei, 20 


\section{Conference Committees}

Honorary Chairs

Xun Hou, Chinese Academy of Sciences (China)

Zhuangde Jiang, Chinese Academy of Engineering (China)

General Chairs

Chunmin Zhang, Xi'an Jiaotong University (China)

Wei Zhao, Xi'an Institute of Optics and Precision Mechanics (China)

Weiguo Liu, Xi' an Technological University (China)

Dongxu Cui, Xi' an Institute of Applied Optics (China)

Anand Asundi, Nanyang Technological University (Singapore)

Secretary

Feng Chen, Xi'an Jiaotong University (China)

Deputy Secretary

Piao Rong, Xi'an Jiaotong University (China)

Academic Committee Chairs

Songlin Zhuang, Chinese Academy of Engineering (China)

Qingxi Tong, Chinese Academy of Sciences (China)

Wenqing Liu, Chinese Academy of Engineering (China)

Qihuang Gong, Chinese Academy of Sciences (China)

Yongqi Xue, Chinese Academy of Sciences (China)

Huixing Gong, Chinese Academy of Engineering (China)

Theo Tschudi, Technische Universität Darmstadt (Germany)

Academic Committee Members

Anand Asundi, Nanyang Technological University (Singapore)

Jintao Bai, Northwest University (China)

Guangde Chen, Xi'an Jiaotong University (China)

Christian von Savigny, Emst-Moritz-Arndt-Universität Greifswald (Germany)

Yixiang Duan, Sichuan University (China)

Xuewu Fan, Xi' an Institute of Optics and Precision Mechanics (China)

Hong Gao, Xi'an Jiaotong University (China)

Junjie Guo, Xi'an Jiaotong University (China) 
Jun Han, Xi'an Technological University (China)

Bingliang Hu, Xi'an Institute of Optics and Precision Mechanics

(China)

Ming Ji, Xi' an Institute of Applied Optics (China)

Fuli Li, Xi' an Jiaotong University (China)

Qinxue Li, Xi' an Institute of Applied Optics (China)

Yan Li, Peking University (China)

Wuming Liu, Institute of Physics, Chinese Academy of Sciences (China)

Qingming Luo, Huazhong University of Science and Technology

(China)

Mitsuo Takeda, Utsunomiya University (Japan)

Xueguang Qiao, Northwest University (China)

Kuanfang Ren, Université Roven (France)

Xiaopeng Shao, Xidian University (China)

Guangming Shi, Xidian University (China)

Tobias Haist, Universität Stuttgart (Germany)

Dingyi Wang, University of New Brunswick (Canada)

Wei Wang, Herot Watt University (United Kingdom)

Xiao Wang, China Academy of Engineering Physics (China)

Zhaomin Wang, Nanyang Technological University (Singapore)

Desheng Wen, Xi' an Institute of Optics and precision Mechanics

(China)

Zhiyu Wen, Chongqing University (China)

William E. Ward, University of New Brunswick (Canada)

Wolfgang Osten, Universität Stuttgart (Germany)

Guang Xi, Xi'an Jiaotong University (China)

Jianfeng Yang, Xi' an Institute of Optics and Precision Mechanics

(China)

Baoli Yao, Xi'an Institute of Optics and Precision Mechanics (China)

Hairong Zheng, Shaanxi Normal University (China)

Jianlin Zhao, Northwestern Polytechnical University (China)

Weihu Zhou, Institute of Opto-electronics, Chinese Academy of

Sciences (China)

Yuquan Zheng, Chinese Academy of Sciences (China)

Zhenxi Zhang, Xi'an Jiaotong University (China)

Organizing Committee

Anand Asundi, Nanyang Technological University (Singapore)

Feng Chen, Xi' an Jiaotong University (China)

Dongxu Cui, Xi' an Institute of Applied Optics (China)

Guangqing Du, Xi'an Jiaotong University (China)

Li Liang, Xi'an Jiaotong University (China)

Weiguo Liu, Xi'an Technological University (China)

Zhe Qu, Xi' an Jiaotong University (China) 
Jiangbo She, Xi' an Institute of Optics and Precision Mechanics

(China)

Jianhua Sun, Huanic Corporation (China)

Ailing Tian, Xi'an Technological University (China)

Wei Wu, Xi'an Institute of Applied Optics (China)

Weiliang Yan, Xi'an Institute of Applied Optics (China)

Xiaoxu Yang, Xi'an Institute of Optics and Precision Mechanics (China)

Chunmin Zhang, Xi'an Jiaotong University (China)

Wei Zhao, Xi' an Institute of Optics and Precision Mechanics (China)

Renkui Zhou, Xi'an Institute of Optics and Precision Mechanics

(China)

Xueliang Zhu, Xi'an Technological University (China)

Program Committee

Hao Bian, Xi' an Jiaotong University (China)

Feng Chen, Xi'an Jiaotong University (China)

Guangqing Du, Xi'an Jiaotong University (China)

Dangqi Fang, Xi'an Jiaotong University (China)

Yongyi Huang, Xi' an Jiaotong University (China)

Qiwei Li, Xi'an Jiaotong University (China)

Dongdong Liu, Xi' an Jiaotong University (China)

Yanfen Li, Xi' an Jiaotong University (China)

Ruifeng Liu, Xi' an Jiaotong University (China)

Shengli Ma, Xi'an Jiaotong University (China)

Tingkui Mu, Xi' an Jiaotong University (China)

Wenyi Ren, Northwest A\&F University (China)

Piao Ring, Xi' an Jiaotong University (China)

Jiangbo She, Xi' an Institute of Optics and Precision Mechanics

(China)

Ailing Tian, Xi'an Technological University (China)

Tingyu Yan, Xi' an Jiaotong University (China)

Jirui Zhang, Xi' an Jiaotong University (China)

Zhaoyu Zuo, Xi' an Jiaotong University (China) 
Proc. of SPIE Vol. 10256 1025601-22 Downloaded From: https://www.spiedigitallibrary.org/conference-proceedings-of-spie on 26 Apr 2023
Terms of Use: https://www.spiedigitallibrary.org/terms-of-use 


\section{Introduction}

Following the success of icPOE2014, the Second International Conference on Photonics and Optical Engineering (icPOE2016) was successfully hosted 14-17 October 2016, in Xi'an China.

The field of photonics and optical engineering is developing rapidly worldwide. Related technologies have been applied in both Space and Mars exploration, micro-nano devices and new materials development, biomedicine, quantum computers, and quantum communication. The "National Guideline on Medium and Long Term Program for Science and Technology Development (2006-2020)" of China, has multiple priority areas and great demands that mention photonics and optical engineering involving remote sensing, environmental monitoring, biomedical diagnosis, space exploration, astronomy, and other scientific areas.

At present, the world's largest radio telescope, FAST (Five-hundred-meter Aperture Spherical radio Telescope), will be put into use; the Chang'e IV lunar probe will also launch for exploration of the back of the moon; and a Mars exploration program has been formally established and its exploration satellite will be launched around 2020. These are a concrete embodiment of the rapid development of China's photonics and optical engineering achievements.

Indeed, there have been significant advances as well over the past years. There are an increasing number of photonics and optical engineering companies setting up or ramping up their manufacturing facilities here, and at the same time, some home grown companies are show-casing innovations and technologies. In order to cater to these developments, many universities and scientific research institutions in Xi'an, Shanghai, Tianjin, and other famous cities have established 'Photonics Research Centres'. These institutions have organized many national academic conferences for deeper academic exchanges and scientific interactions, as well as to strengthen the cultivation of excellent talent, and to reach out to the younger generation to highlight career and research options in photonics and optical engineering.

The International Conference on Photonics and Optical Engineering (icPOE) is one of the advanced academic forums in the world. The purpose of this conference is to build an international communication platform for optical research to aim at the opportunity of emerging markets and technology and to strengthen the advanced technology of optical application and measurement for innovation and development through a series of academic exchanges. This conference is significant in promoting communication of the latest achievements, enhancing mutual understanding of the status and trend of developments, and strengthening international cooperation in the optical field. 
Leading scientists and researchers from all over the world submitted 170 papers, which were organized under seven topics:

1. Space optics

2. Spectroscopy and applications

3. Ultrafast optics

4. Photonic functional materials and integrated photonic device

5. Optical design and manufacturing technology

6. Optical measurement and inspection

7. Micro-nano manufacturing and testing.

We would like to express our heartfelt thanks to Shaanxi Optical Society, Shaanxi Provincial Physical Society, Optics and Photonics Society of Singapore (OPSS), Chinese Optical Society, SPIE, Xi'an Jiaotong University, Xi'an Institute of Optics and Precision Mechanics of Chinese Academy of Sciences, Xi' an Technological University, Xi'an Institute of Applied Optics, Centre for Optical and Laser Engineering of Nanyang Technological University (Singapore), Ministry of Education of China, Chinese Academy of Sciences, National Natural Science Foundation of China, Shaanxi Association for Science and Technology, other supporting organizations, and the organizing committee members, for their full support of this conference.

Our appreciation also goes to all the authors who spent a lot of time and effort in the preparation of their papers. Our deep thanks are directed to the students Rong Piao, Li Qiwei, Yan Tingyu, Liu Dongdong, Zhang Jirui, Li Yanfen, Gao Meng, Quan Naicheng, Chen Zeyu, Zhang Guoqing, Jiao Zuzu, and Wu Miao, and the volunteers Zhang Biyun, Zhang Fan, Wei Yang, Li Wentao, Zhang Hong, Zhao Siwei, and Yang Chen. The continuous help given especially by Yang Xiaoxu, Du Guangqing, Zhu Xueliang and She Jiangbo was the basis for making icPOE2016 successful.

Finally, our special thanks and appreciation goes to all of our friends and colleagues for sharing with us the positive results of photonics and optical engineering. 